\title{
Influence of a Low-Level Semiconductor Gallium Arsenate Laser in Experimental Envenomation Induced by Bothrops atrox Snake Venom
}

\author{
${ }^{1}$ Elziliam Aranha De Sousa, ${ }^{1}$ José Adolfo Homobono Machado Bittencourt, \\ ${ }^{1}$ Nayana Keyla Seabra De Oliveira, ${ }^{1}$ Shayanne Vanessa Correia Henriques, \\ ${ }^{1}$ Leide Caroline Dos Santos Picanço, ${ }^{1}$ Camila Pena Lobato, ${ }^{2}$ José Renato Ribeiro, \\ ${ }^{3}$ Washington Luiz Assunção Pereira, ${ }^{4}$ José Carlos Tavares Carvalho, ${ }^{1}$ Jocivânia Oliveira Da Silva \\ ${ }^{1}$ Laboratório de Toxicologia, Curso de Ciências Farmacêuticas, \\ Universidade Federal do Amapá, Macapá, AP, Brazil \\ ${ }^{2}$ Serviço de Zoonoses, Secretaria de Estado da Saúde, Macapá, AP, Brazil \\ ${ }^{3}$ Laboratório de Patologia Animal, Universidade Federal Rural da Amazônia, Belém, PA, Brazil \\ ${ }^{4}$ Laboratório de Pesquisa em Fármacos, \\ Curso de Ciências Farmacêuticas, Universidade Federal do Amapá, Macapá, AP, Brazil
}

Received 2012-11-06, Revised 2012-12-28; Accepted 2012-12-29

\begin{abstract}
Bothrops atrox is responsible for the majority of snakebite accidents in Brazilian Amazon and its venom can cause prominent local tissue damage. Experimental groups consisted of five male mice, each administered either B. atrox Venom (VB), B. atrox Venom + Antivenom (VAV), B. atrox Venom + Laser (VL), B. atrox Venom + Antivenom + Laser (VAVL), or Sterile Saline Solution (SSS) alone. Paw oedema was induced by intradermal administration of $0.05 \mathrm{mg} \mathrm{kg}^{-1}$ of $B$. atrox venom and was expressed in mm of directly induced oedema. Mice were subcutaneously injected with $0.10 \mathrm{mg} \mathrm{kg}^{-1}$ of venom for evaluation nociceptive activity and the time (in seconds) spent in licking and biting responses of the injected paw were taken as an indicator of pain response. Inflammatory infiltration was determined by counting the number of leukocytes present in the gastrocnemius muscle after venom injection $\left(0.10 \mathrm{mg} \mathrm{kg}^{-1}\right)$. Myotoxicity was studied by determining the plasmatic rise of creatine kinase activity after venom injection $\left(0.20 \mathrm{mg} \mathrm{kg}^{-1}\right)$. For histological examination of myonecrosis, venom $\left(0.10 \mathrm{mg} \mathrm{kg}^{-1}\right)$ was administered intramuscularly. The site of venom injection was irradiated by GaAs laser and some animals received antivenom intraperitoneally. GaAs laser irradiation administered in conjunction with antivenom, reduced pain, oedema, inflammation and myonecrosis induced by $B$. atrox venom in mice. The combined antivenom and GaAs laser treatment was more effective than separately treatments. The results suggest that laser therapy may reduce the local effects induced by $B$. atrox venom when associated with antivenom.
\end{abstract}

Keywords: GaAs (Gallium Arsenate) Laser, Bothrops Atrox, Snake Venom

\section{INTRODUCTION}

Envenoming resulting from snake bites is a particularly important public health problem in Amazon Brazilian. Significant numbers of these accidents occur annually and generally are caused by Bothrops snake. Difficulties in access to health services, delay medical care and snakes specimens unidentified difficult treatments of envenomation cases, which are usually prescribed anti-Bothrops and Lachesis serum.
At present, adequate treatment of snakebite envenoming, depend on the ability of antivenoms to reverse signs systemic as coaguloapty, hemorrhage, hypontensive shock and others disorders (Calvete et al., 2009), which neutralizes systemic alterations, but does not prevent local effects and resultant disabilities (Nascimento et al., 2010). Other therapeutic alternatives have been proposed to complement the treatment of local effects caused by bothropic venom, such as the use of plant extracts (Silva et al., 2005),

Corresponding Author: Elziliam Aranha de Sousa, Laboratório de Toxicologia, Curso de Ciências Farmacêuticas, Universidade Federal do Amapá, Macapá, AP, Brazil 
synthetic drugs (Lomonte et al., 2009) and low power laser (Barbosa et al., 2008).

The low power laser acts on the microvasculature, acting on the precapillary sphincter, causing vasodilation and reabsorption of exudate and the bloodstream, increasing the speed of movement. Also serves on the fibrinolytic system, favoring the elimination of microthrombi. In addition, causing an increase in phagocytosis by increasing the number of macrophages and oxygen, acting as biomodulator or normalizing cell (Díaz et al., 2009).

For these reasons, the aim of this study was to investigate whether low-level semiconductor GaAs (Gallium-Arsenate) laser irradiation, applied locally at different intervals of time, after $B$. atrox venom and antivenom administration in mice, could be alternative methods to minimize local damage produced by snakebite. The data provided also may contribute to understand the laser therapy effects, as well as to determine the number of applications and period of irradiation in envenoming cases by Bothrops snake.

\section{MATERIALS AND METHODS}

\subsection{Low-Level Laser}

A low-level semiconductor GaAs laser from HTM Compact (Amparo, Sao Paulo, Brazil) operating at a wavelength of $904 \mathrm{~nm}$ was used. The laser parameters were configured to $50 \mathrm{~mW}$ powers, an irradiation time of $25 \mathrm{sec}$ and an irradiated area $2 \mathrm{~cm}^{2}$ at a dose of $4 \mathrm{~J} / \mathrm{cm}^{2}$. Laser irradiation was performed by the direct contact of the stylus at an angle perpendicular to the skin at the exact area where the venom was administered. The laser apparatus was calibrated using an Ophir Pulsar Power Optical Meter. The dose used in the experiments (4 $\mathrm{J} / \mathrm{cm}^{2}$ ) does not cause thermal effects and its beneficial effects have been cited in several studies. These parameters were used in all experiments.

\subsection{Venom and Antivenom}

B. atrox venom was supplied from the Serpentarium at the Laboratório de Toxicologia, Curso de Ciências Farmacêuticas, Universidade Federal do Amapá, Amapá, Brazil. The venom was lyophilized and kept under refrigeration at $4^{\circ} \mathrm{C}$. The venom was diluted in apyrogenic saline solution $(0.9 \%)$ immediately prior to its use. The antivenom, which was obtained from the Instituto Vital Brazil, Rio de Janeiro, Brazil, was a polyvalent bothropic and lachesis antivenom (Lot MS 095904 B) provided by Central de Armazenamento e Distribuição de Imunobiológicos, Secretaria de Estado da Saúde, Amapá, Brazil. This antivenom is raised against a pool of venoms from Bothrops jararaca, Bothrops jararacussu, Bothrops alternatus, Bothrops moojeni, Bothrops neuwiedi and Lachesis muta.

\subsection{Animals}

All animal care was performed in accordance with the guidelines of the Brazilian College for Animal Experimentation. Male Swiss Webster mice weighing 20$25 \mathrm{~g}$ were used for the experiments and were randomly divided into groups of five animals each. The mice were kept in plastic cages with access to water and food ad libitum and were maintained under controlled temperatures $\left(18-20^{\circ} \mathrm{C}\right)$ on a $12 \mathrm{~h}$ light/dark cycle.

\subsection{Groups and Experimental Protocols}

The experimental groups consisted of five male mice each administered $B$. atrox Venom (VB) alone, $B$. atrox Venom + Antivenom (VAV), B. atrox Venom + Laser (VL), B. Atrox Venom + Antivenom + Laser (VAVL), or Sterile Saline Solution (SSS) alone. All the venom solutions used in the experiments were diluted in $50 \mu \mathrm{L}$ of a sterile saline solution prior to injection. The animals were immobilised manually and the laser device was applied by contact in the same location as the injection of venom or saline solution. The venom doses used were selected from previous dose-response experiments, in which it was observed that the venom induced a minimum response for all activities evaluated. Sixty microliters of polyvalent bothropic and lachesis antivenom were injected intraperitoneally (i.p.) $30 \mathrm{~min}$ after venom administration (Nadur-Andrade et al., 2012).

\subsection{Oedema Induction}

The minimum dose was defined as the lowest venom dose required for the formation of $30 \%$ paw oedema (Rocha and Furtado, 2007). To measure paw oedema, $0.05 \mathrm{mg} \mathrm{kg}^{-1}$ of B.atrox venom was diluted in $50 \mu \mathrm{L}$ of a sterile saline solution and administered intradermally into the subplantar region in mice. Laser administration was performed $30 \mathrm{~min}$ after venom injection and immediately after antivenom injection. The laser irradiation was repeated at 1, 6, 24, 30, 48,54 and $72 \mathrm{~h}$. The animals of the control group received only $50 \mu \mathrm{L}$ of sterile saline solution in the subplantar region. Paw oedema was measured with the aid of a low-pressure pachymeter (Mitutoyo, Japan) and is expressed in $\mathrm{mm}$ of directly induced oedema.

\subsection{Induction of Nociception}

The method used was a modification of the method previously described by Hunskaar and Hole (1987). Sample containing $0.05,0.10$ or $0.20 \mathrm{mg} \mathrm{kg}^{-1}$ of venom in $50 \mu \mathrm{L}$ of a sterile saline solution were injected subcutaneously for evaluated venom nociceptive effect. The minimum dose was defined as the lowest venom dose required for a statistically significant increase $(\mathrm{p}<0.05)$ at time (in seconds) spent in licking and biting responses of the injected paw. Effects of laser and antivenom were evaluated after $B$. atrox venom 
administration $\left.(0.10 \mathrm{mg} \mathrm{kg})^{-1}\right)$ diluted in $50 \mu \mathrm{L}$ of a sterile saline solution subcutaneously into the right hind paw. VAV group animals received antivenom intraperitoneally immediately after venom injection. Laser was applied in VAVL group animals immediately after venom and antivenom administration. The animals of the control group were administered $50 \mu \mathrm{L}$ of sterile saline solution subcutaneously. The mice were then put back in the chamber with mirrors and the observation period started. Distinct periods of intensive licking activity were identified and scored separately unless otherwise stated. The first period (early phase) was recorded 0-5 min and the second period (late phase) was recorded 20-30 min after the injection in the corresponding groups. The time (in seconds) spent in licking and biting responses of the injected paw were taken as an indicator of pain response. The test was performed at ambient temperature of $22-26^{\circ} \mathrm{C}$ and care was taken to exclude environmental disturbances (high temperature, noise and excessive movement) that might interfere in the study.

\subsection{Myotoxic Activity}

Myotoxicity was studied by determining the plasmatic levels of creatine kinase activity (Gutiérrez et al., 1980) after intramuscular injection of the venom. $B$. atrox venom $\left(0.20 \mathrm{mg} \mathrm{kg}{ }^{-1}\right)$ was diluted in $50 \mu \mathrm{L}$ of a sterile saline solution and administered into the gastrocnemius muscle. VAV group animals received antivenom intraperitoneally immediately after venom injection. Laser was applied in VAVL group animals immediately after venom and antivenom administration. The animals of the control group were administered with $50 \mu \mathrm{L}$ of sterile saline solution i.m. After $3 \mathrm{~h}$ blood was collected from the orbital plexus into heparinized capillary tubes and the plasma creatine kinase activity was determined by a kinetic assay (CK NAC UV $\mathrm{K} 010$ ). Activity was expressed in U/l, one unit defined as the phosphorylation of $1 \mathrm{mmol}$ of creatine $/ \mathrm{min}$ at $37^{\circ} \mathrm{C}$.

\subsection{Quantification of Inflammatory Infiltrate in Muscle}

Inflammatory activity assays were performed as previously described (Barbosa et al., 2008; Teixeira et al., 2003). B. atrox venom $\left(0.10 \mathrm{mg} \mathrm{kg}^{-1}\right)$ diluted in $50 \mu \mathrm{L}$ of a sterile saline solution was administered in the right gastrocnemius muscle of mice. Thirty min later, the laser device was applied by contact in the same location as the injection of venom. Laser irradiation was repeated at 1, 6, $24,30,48,54$ and $72 \mathrm{~h}$. The animals of the control group received only $50 \mu \mathrm{L}$ of sterile saline solution intramuscularly (i.m.). The mice were euthanised after $72 \mathrm{~h}$ in a CO 2 chamber. The gastrocnemius muscles were collected and cut with a blade into very small pieces before the addition of $2 \mathrm{~mL}$ of $\mathrm{PBS}$. The suspension was incubated for $30 \mathrm{~min}$ at $4^{\circ} \mathrm{C}$, after which it was filtered through gauze. A fraction of the filtered solution was diluted in liquid of Turk $(1: 20)$ for the total counting of the leukocytes in a Neubauer chamber.

\subsection{Histological Examination of Myonecrosis}

B. atrox venom $\left(0.10 \mathrm{mg} \mathrm{kg}{ }^{-1}\right.$ in $50 \mu \mathrm{L}$ of a sterile saline solution) was administered i.m. in the right gastrocnemius muscle. Laser administration was performed $30 \mathrm{~min}$ after venom injection and immediately after antivenom injection. The laser application was repeated at 1, 6, 24, 30, 48, 54 and $72 \mathrm{~h}$. The animals of the control group received only 50 $\mu \mathrm{L}$ of sterile saline solution i.m. The mice were euthanised after $72 \mathrm{~h}$ in a $\mathrm{CO} 2$ chamber. The gastrocnemius muscles were collected for histological processing, as described previously by Santos-Filho and coworkers (Jorge et al., 1999).

\subsection{Statistical Analysis}

The results are presented as the mean \pm S.E.M. Differences among groups were analyzed by one-way Analysis of Variance (ANOVA) followed by TukeyKramer test. Differences with an associated probability (P value) of less than $5 \%(p<0.05)$ were considered significant.

\section{RESULTS}

\subsection{Inhibition of Oedema Induced by B. Atrox Venom}

Oedema volume was measured at times 1, 2, 3, 4, 6, $24,30,48,54$ and $72 \mathrm{~h}$ after treatments. Fig. 1 shows the effect of venom, GaAs laser and antivenom in the edema formation in mice. At the end $72 \mathrm{~h}$, it can be observed significant reduction of paw edema in the VAVL and VAV groups when compared to VB group animals administered only with $B$. atrox venom. Treatment of the animals only with laser (VL group) was shown to be ineffective in reducing the paw edema.

\subsection{Evaluation of Antivenom and Laser Treatment on Nociceptive Activity of the $B$. Atrox Venom}

The subcutaneous injection of $0.10 \mathrm{mg} \mathrm{kg}^{-1} B$. atrox venom into the right hind paw caused licking and biting responses in injected local as an indicator of pain response. In the first phase of the nociceptive activity (Fig. 2A), the results in all test groups were not significantly different from VB control group. As observed in Fig. 2B (second phase), only the VAVL group $(31.6 \pm 21.5, p<0.05)$ showed a reduction in the time (in seconds) spent licking and biting the injected paw compared to the VB group (98 \pm 7.3$)$.

\subsection{Effect of GaAs Laser and Antivenom on the in vivo Myotoxicity Induced by $B$. Atrox Venom in Mice}

Intramuscular injections of $B$. atrox crude venom $(0.2$ $\mathrm{mg} \mathrm{kg}^{-1}$ ) induced a significant increase on plasma CK activity when compared to SSS control group from 156.5 $\pm 83.05-1646 \pm 675.3,3 \mathrm{~h}$ after venom injection (Fig. 3). 


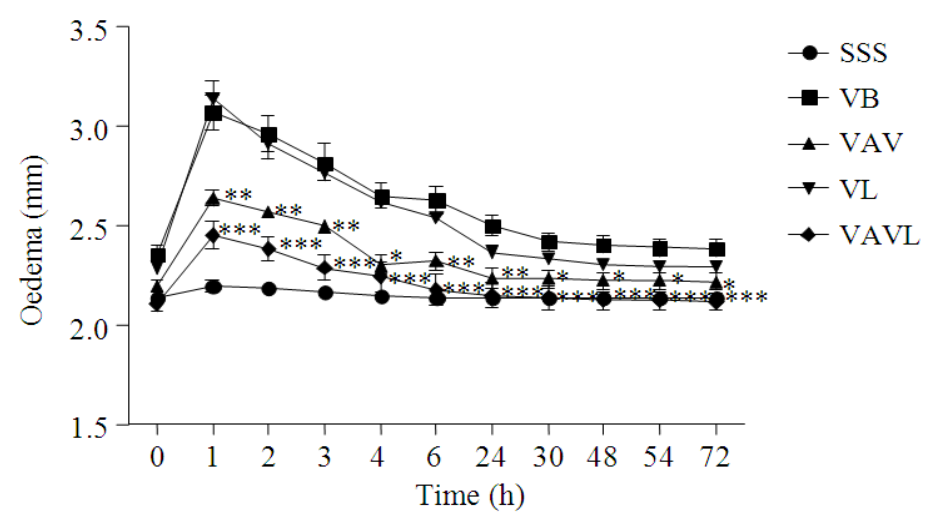

Fig. 1. Effect of the gallium arsenate laser $\left(4 \mathrm{~J} / \mathrm{cm}^{2}\right)$ on oedema induced by $B$. atrox venom, administered i.d. in the subplantar region $(0.05$ $\mathrm{mg} \mathrm{kg}^{-1}$ of venom). Laser administration was performed $30 \mathrm{~min}$ after venom injection. In the animals that received antivenom, irradiation occurred immediately after the application of antivenom. The laser application was repeated at 1, 6, 24, 30, 48, 54 and 72 $\mathrm{h}$. The results are presented as the mean \pm S.E.M. of five animals. Differences among groups were analysed by one-way Analysis Of Variance (ANOVA), followed by the Tukey-Kramer test. Differences with an associated probability (p value) of less than $5 \%$ $(\mathrm{p}<0.05)$ were considered significant. ${ }^{*} \mathrm{p}<0.05,{ }^{* *} \mathrm{p}<0.01,{ }^{* * *} \mathrm{p}<0.001$ compared with VB group

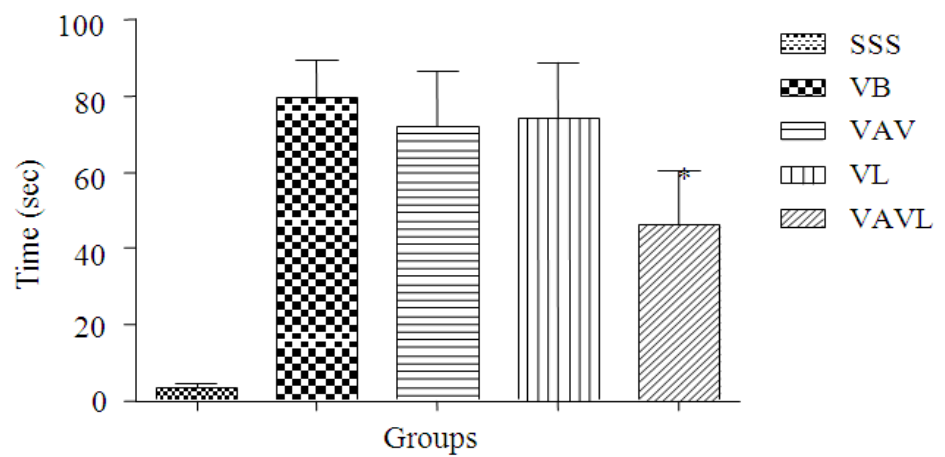

(A)

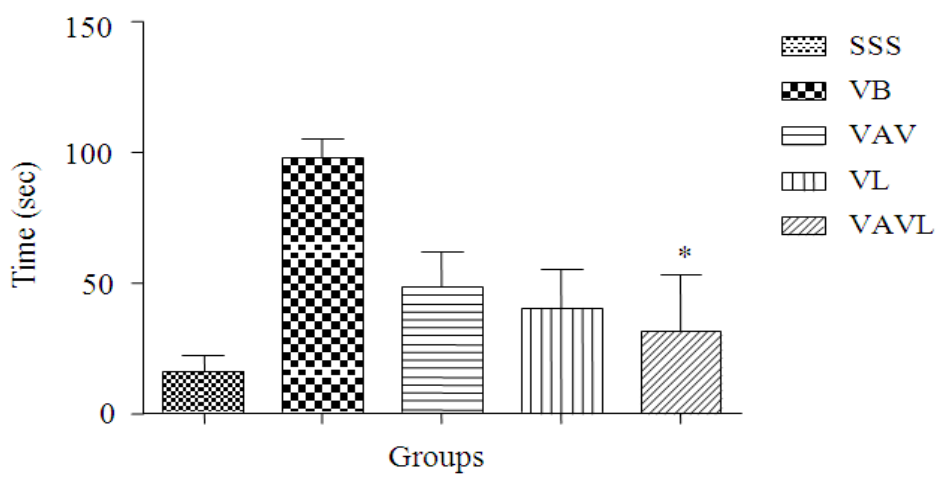

(B)

Fig. 2. Effect of the gallium arsenate laser $\left(4 \mathrm{~J} / \mathrm{cm}^{2}\right)$ on the nociceptive activity induced by B. atrox venom $\left(0.10 \mathrm{mg} \mathrm{kg}{ }^{-1}\right)$ injected subcutaneously into the right hind paw. The animals of the control group were given $50 \mu \mathrm{L}$ of sterile saline solution subcutaneously. The responses were measured at $5 \mathrm{~min}$ (first phase-A) and 20-30 $\mathrm{min}$ (second phase-B) after venom or saline solution administration. The results are presented as the mean \pm S.E.M. of five animals. Differences among groups were analysed by one-way Analysis Of Variance (ANOVA), followed by the Tukey-Kramer test. Differences with an associated probability ( $p$ value) of less than $5 \%(p<0.05)$ were considered significant. ${ }^{*} p<0.05$, compared with VB group 


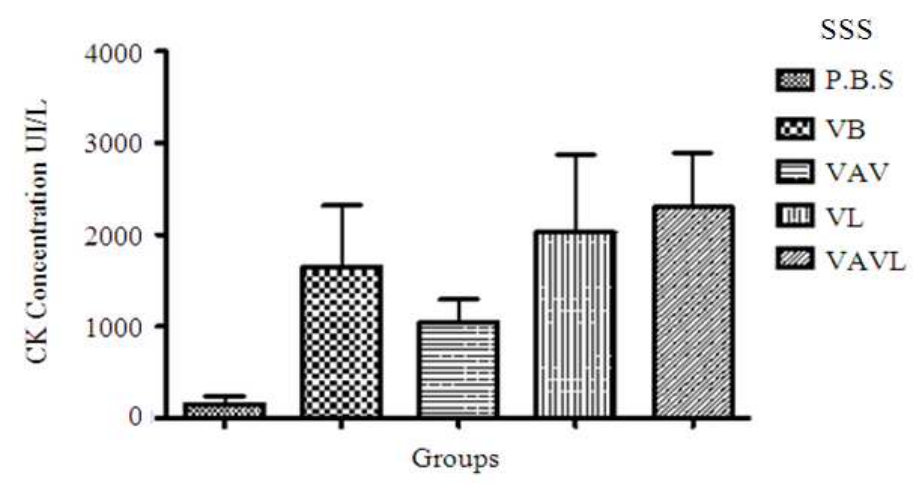

Fig. 3. Effect of the gallium arsenate laser $\left(4 \mathrm{~J} / \mathrm{cm}^{2}\right)$ on the myotoxicity induced by $B$. atrox venom $\left(0.20 \mathrm{mg} \mathrm{kg}^{-1}\right)$ injected intramuscular route into the gastrocnemius muscle. The animals of the control group were given $50 \mu \mathrm{L}$ of sterile saline solution i.m.. After $3 \mathrm{~h}$ blood was collected from the orbital plexus into heparinized capillary tubes and the plasma creatine kinase activity was determined by a kinetic assay (CK NAC UV K010). The results are presented as the mean \pm S.E.M. of five animals. Differences among groups were analysed by one-way Analysis Of Variance (ANOVA), followed by the Tukey-Kramer test

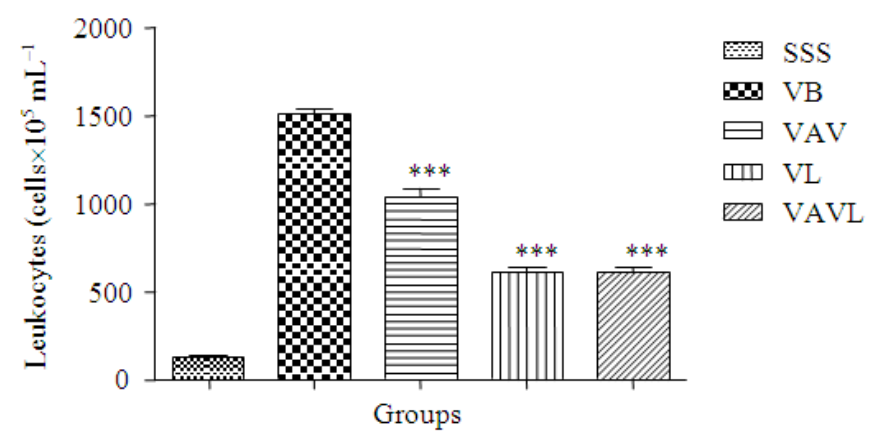

Fig. 4. Inflammatory infiltration induced by the $B$. atrox venom and gallium arsenate laser treatment $\left(4 \mathrm{~J} / \mathrm{cm}^{2}\right)$. The mice were injected with $0.10 \mathrm{mg} \mathrm{kg}^{-1}$ B. atrox venom in the right gastrocnemius muscle. The laser device was applied by contact in the same local region of the injection $30 \mathrm{~min}$ after the administration of venom. The laser application was repeated at 1, 6, 24, 30, 48, 54 and $72 \mathrm{~h}$. The animals of the control group received only $50 \mu \mathrm{L}$ of sterile saline solution via intramuscular (i.m.) injection. The results are presented as the mean \pm S.E.M. of five animals. Differences among groups were analysed by one-way Analysis Of Variance (ANOVA), followed by the Tukey-Kramer test. Differences with an associated probability ( $p$ value) of less than $5 \%(p<0.05)$ were considered significant. ${ }^{* * *} p<0.001$ compared with VB group

The results also showed that GaAs laser and antivenom (VAV, VL and VAVL groups) were not able to inhibit increase on plasma CK activity when compared to VB control group.

\subsection{Local inflammatory Infiltrate Induced by $B$. Atrox Venom Administration and
Antivenom and Laser Treatment}

In our experimental model, B. atrox venom was intramuscularly administered and the laser applied by contact in the same location as the injection of venom, 30 min, 1, 6, 24, 30, 48, 54 and $72 \mathrm{~h}$ later (Fig. 4). Inflammatory infiltrate was determined by counting the number of leukocytes present in the gastrocnemius muscle. The total counting of these cells showed that $B$. atrox venom $\left(0.10 \mathrm{mg} \mathrm{kg}{ }^{-1}\right)$ induced increase in the number of the leukocytes in VB group $(1510 \pm 33.1)$. After $72 \mathrm{~h}$, a statistically significant reduction in the total number of leukocytes was observed for the VAV, VL and VAVL groups $(1040 \pm 50.1, \mathrm{p}<0.001 ; 610 \pm 24.4$, $\mathrm{p}<0.001 ; 610 \pm 24.4, \mathrm{p}<0.001$, respectively).

\subsection{Histological Examination of Myonecrosis after B. Atrox Venom Administration and antIvenom and Laser Treatment}

The gastrocnemius muscles of the animals that received only sterile saline solution were histologically normal, with normal conjunctiva and myocytes and without any evidence of inflammatory or degenerative alterations. 


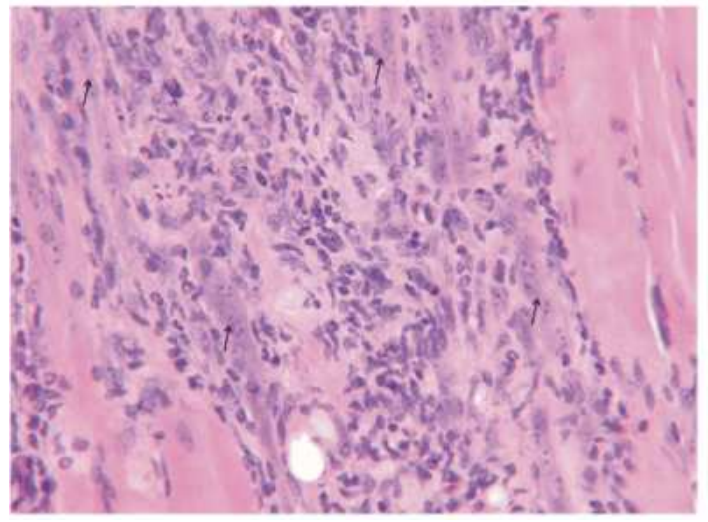

(A)

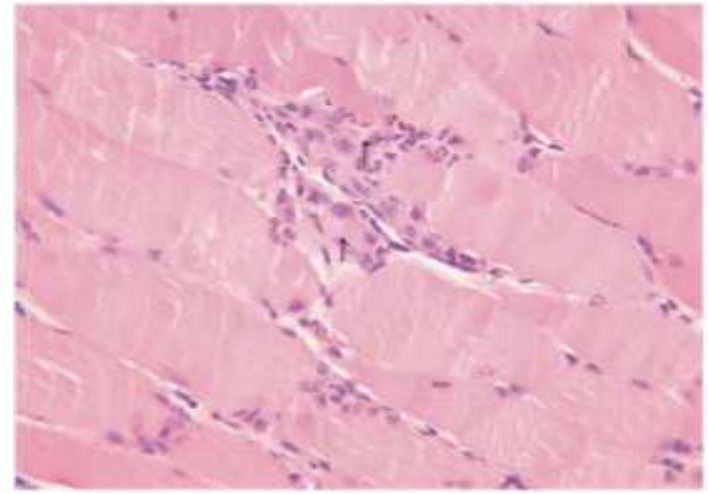

(C)

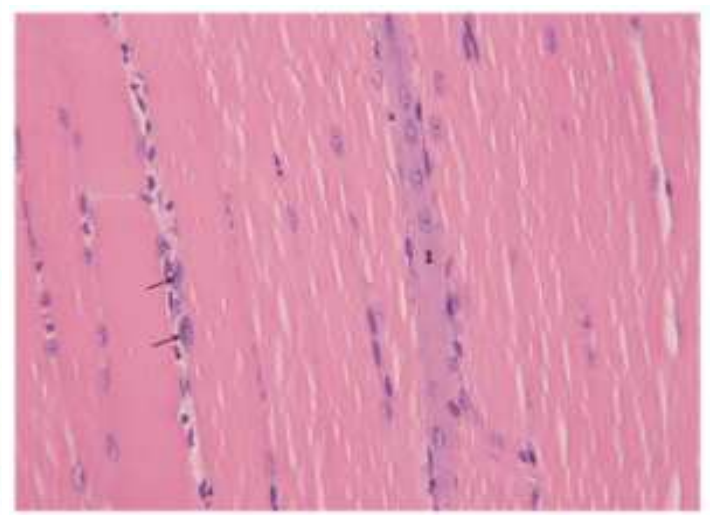

(B)

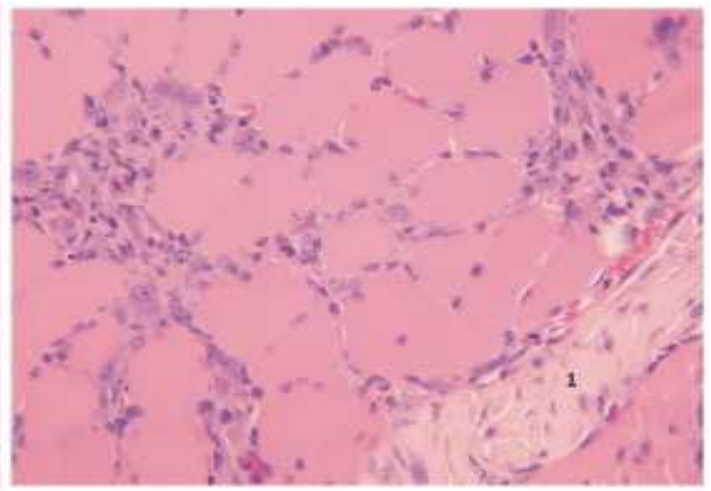

(D)

Fig. 5. Histopathological observations of the gastrocnemius muscle of mice $72 \mathrm{~h}$ after exposure to $B$. atrox venom. (A) Photomicrograph of the gastrocnemius muscle of animals given B. atrox venom. Muscle area with reaction infiltrate of macrophages and some neutrophils in the presence of myocyte regeneration (arrows) were observed. (B) Photomicrograph of the gastrocnemius muscle of animals administered $B$. atrox venom + laser irradiation. Regeneration of isolated muscle fiber (1) and reaction of satellite cells (arrows) were observed. (C) Photomicrograph of the gastrocnemius muscle of animals given B. atrox venom + antivenom. Alterations perimysium of muscle, with a dense infiltration. Damage segmental, regeneration of myocytes (arrows). (D) Photomicrograph of the gastrocnemius muscle of animals administered B. atrox venom+ serum + laser irradiation. The perimysium shows up with normal (1). In cross sectional, we observe areas of muscle reaction that involves banks of regenerating fibers perimysium. Haematoxylin/Eosin (HE)

The mice given $B$. atrox venom alone, after $72 \mathrm{~h}$, showed inflammatory process with loss of muscle fibers, infiltrate of macrophages and polymorphonuclear cells (neutrophils) (Fig. 5A). Other alterations had occurred, with proliferation of satellite cells, indicating regeneration after necrosis. The VL group animals demonstrated perimysium changes of muscles, dense infiltration with macrophages and some neutrophils, fibroblast proliferation, myocites regeneration and did not reveal necrosis (Fig. 5B). The histological analysis of the animals in the VAV group showed segment regeneration of fibers with sarcoplasm denser, basophilic and multicore, perimysium changes of muscles, dense infiltration with macrophages and some neutrophils and fibroblast proliferation, moderate inflammation, with a predominance of neutrophils and macrophages in phagocytosis, some fibers with segmental necrosis (Fig. 5C). After 72 h, in the gastrocnemius muscle of the VAVL group animals, regenerating muscle fibers, necrotic cells in phagocytosis, perimysium changes of muscles and regeneration of some myocytes, was observed (Fig. 5D).

\section{DISCUSSION}

The biological effects of venoms are complex because different components have distinct actions and may, in addition, act in concert with other venom molecules. Snakes of the family Viperidae contain numerous proteins that which can cause local tissue 
necrosis in human victims of envenoming (Fox and Serrano, 2005; Markland, 1998). Antivenom administration initiated rapidly after envenomation, can neutralize systemic effects, but neutralization of local tissue damage is a more difficult task. In a number of snake bite cases, lack of neutralization of local effects results in permanent sequelae, with tissue loss (Jorge et al., 1999. In the current study, the effect of a low-level semiconductor GaAs laser on the local pathological alterations induced by $B$. atrox snake venom was investigated. The effects of low-power lasers are caused by nonthermal mechanisms (King, 1989) produced by light interaction with tissue, resulting in biomodulatory effects through photochemical interactions (Basford, 1995).

Our studies in mice showed that venom-induced paw oedema immediately after administration, gradually decreasing over the $72 \mathrm{~h}$ time period that was assessed. This study corroborates the results of previous studies that indicated the ability of Bothrops venom to induce oedema formation through the action of the venom constituents on vascular endothelial cells and inflammatory mediators (Barbosa et al., 2008). A reduction in the oedematogenic effect induced by $B$. atrox venom was observed for the VAVL and VAV groups after treatment with GaAs laser in the present study. Results obtained by Barbosa et al. (2008), reported the reduction of the oedematogenic effect induced by Bothrops jararacussu snake venom administered intrasmuscularly associated with antivenom (64\% in $24 \mathrm{~h})$ using a different type of low-power Helium-Neon (HeNe) laser generator with a wavelength of $685 \mathrm{~nm}$.

In this study, treatments were not effective in reducing nociceptive activity first stage. In the second phases, the VAVL group showed statistically significant reduction in nociceptive activity. These data suggest that the effects of the combined treatments (antivenom and GaAs laser) can help in reducing pain of snakebite envenomations, a result that corroborates other studies as Barbosa et al. (2008).

The intramuscular injection of $B$. atrox venom increased the basal level of creatine kinase at $3 \mathrm{~h}$. However, antivenom administered by route intraperitoneal and GaAs laser applied immediately after venom and/or antivenom were unable to antagonize the myotoxic effects produced by venom. Serum therapy, which is capable of neutralizing systemic envenoming effects, is less effective in neutralizing the complex severe local syndrome at the site of snakebite (Doin-Silva et al., 2009).

Our results showed that intramuscular injection of $B$. atrox venom $(0.10 \mathrm{mg} \mathrm{kg})$ in mice resulted in a inflammatory response with leukocyte influx in the administration site. The inflammatory reaction is characterized by a series of complex events, including arteriolar vasodilation followed by increased blood flow and venular permeability, plasma exudation and migration of leukocytes to the extravascular space (Sherwood and Toliver-Kinsky, 2004). Antivenom and GaAs laser treatments were effective in reducing local inflammation induced by $B$. atrox venom. Recently, some studies have demonstrated the efficacy of laser treatment in reducing inflammation and myonecrosis induced by Bothrops venom (Nadur-Andrade et al., 2012; Doin-Silva et al., 2009). Others investigators demonstrated that inflammatory responses can be normalised or reduced by the photochemical effects of laser irradiation. The anti-inflammatory effect of the laser is a result of an increase in tissue metabolism and homeostasis normalisation or increases in cGMP formation by endothelial cells and consequent vasodilation, suggesting that there is also a systemic effect of laser irradiation affecting the inflammation reaction (Campana et al., 1998; Michlovitz, 1990).

In our study, histological analysis results indicated that antivenom treatment in conjunction with the GaAs laser application was more effective than other treatments due most of the muscle fibers visualized showed a regeneration stage.

\section{CONCLUSION}

The results of this study indicate that GaAs laser irradiation operating at a wavelength of $904 \mathrm{~nm}$, with a dose of $4 \mathrm{~J} / \mathrm{cm}^{2}$ administered in conjunction with antivenom, reduced pain, oedema, inflammation and myonecrosis induced by $B$. atrox venom in mice. The combined antivenom and GaAs laser treatment was more effective than separately treatments. In conclusion, the laser therapy effect in reducing local damage induced by $B$. atrox venom may be clinically relevant when associated with antivenom.

\section{ACKNOWLEDGEMENT}

The reasearchers thank Instituto Evandro Chagas, Central de Armazenamento e Distribuição de Imunobiológicos do Amapá and Instituto de Pesquisas Científicas e Tecnológicas do Amapá for their contributions.

\section{REFERENCES}

Barbosa, A.M., A.B. Villaverde, L. Guimaraes-Souza, W. Ribeiro and J.C. Cogo et al., 2008. Effect of low-level laser therapy in the inflammatory response induced by Bothrops jararacussu snake venom. Toxicon, 51: 1236-1244. DOI: 10.1016/j.toxicon.2008.02.007 
Basford, J.R., 1995. Low intensity laser therapy: Still not an established clinical tool. Lasers Surg. Med., 16: 331-342. DOI: $10.1002 / 1 \mathrm{sm} .1900160404$

Calvete, J.J., L. Sanz, Y. Angulo, B. Lomonte and J.M. Gutierrez, 2009. Venoms, venomics, antivenomics. FEBS Lett., 583: 1736-1743. DOI: 10.1016/j.febslet.2009.03.029

Campana, V., M. Moya, A. Gavotto, H. Juri and J.A. Palma, 1998. Effects of diclofenac sodium and He: $\mathrm{Ne}$ laser irradiation on plasmatic fibrinogen levels in inflammatory processes. J. Clin. Laser Med. Surg., 16: 317-320. PMID: 10204437

Silva, J.O.D., J.S. Coppede, V.C. Fernandes, C.D. SantAna and F.K. Ticli et al., 2005. Antihemorrhagic, antinucleolytic and other antiophidian properties of the aqueous extract from Pentaclethra macroloba. J. Ethnopharmacol., 100: 145-152. DOI: 10.1016/j.jep.2005.01.063

Díaz, A.H., B.M.G. Mendez, A.O. Molina, J.L.M. Gil and J.B. Tejeda, 2009. Láser de baja potencia en el tratamiento de las calcificaciones de hombro. Revista De La Sociedade Española Del Dolor, 16: 230-238.

Doin-Silva, R., V. Baranauskas, L. Rodrigues-Simioni and M.A.D. Cruz-Hofling, 2009. The ability of low level laser therapy to prevent muscle tissue damage induced by snake venom. Photochem. Photobiol., 85: 63-69. DOI: 10.1111/j.1751-1097.2008.00397.x

Fox, J.W. and S.M.T. Serrano, 2005. Structural considerations of the snake venom metalloproteinases, key members of the M12 reprolysin family of metalloproteinases. Toxicon, 45: 969-985. DOI: 10.1016/j.toxicon.2005.02.012

Gutiérrez, J.M., F. Chaves and R. Bolanos, 1980. Comparative study of venoms of newborn and adult specimens of bothrops asper. Rev. Biol. Trop., 28: 341-351. PMID: 7323342

Hunskaar, S. and K. Hole, 1987. The formalin test in mice: Dissociation between inflammatory and noninflammatory pain. Pain, 30: 103-104. DOI: 10.1016/0304-3959(87)90088-1

Jorge, M.T., L.A. Ribeiro and J.L. O’Connell, 1999. Prognostic factors for amputation in the case of envenoming by snakes of the Bothrops genus (Viperidae). Ann. Trop. Med. Parasitol., 93: 401408. PMID: 10656041
King, P.R., 1989. Low level laser therapy: A review. Lasers Med. Sci., 4: 141-190. DOI: 10.1007/BF02032427

Lomonte, B., G. Leon, Y. Angulo, A. Rucavado and V. Nunez, 2009. Neutralization of Bothrops asper venom by antibodies, natural products and synthetic drugs: contributions to understanding snakebite envenomings and their treatment. Toxicon, 54: 1012-1028. DOI: 10.1016/j.toxicon.2009.03.015

Markland, F.S., 1998. Snake venoms and the hemostatic system. Toxicon, 36: 1749-1800. DOI: 10.1016/S0041-0101(98)00126-3

Michlovitz, S.L., 1990. Biophysical Principles of Heating and Superficial Heat Agents. In: Thermal Agents in Rehabilitation, S.L Michlovitz, (Ed.). Davis, Philadelphia, pp: 88-107.

Nadur-Andrade, N., A.M. Barbosa, F.P. Carlos, C.J. Lima and J.C. Cogo et al., 2012. Effects of photobiostimulation on edema and hemorrhage induced by Bothrops moojeni venom. Lasers Med. Sci., 27: 65-70. DOI: 10.1007/s10103-011-0914-1

Nascimento, N.G., M.C. Sampaio, R.A. Olivo and C. Teixeira, 2010. Contribution of mast cells to the oedema induced by Bothrops moojeni snake venom and a pharmacological assessment of the inflammatory mediators involved. Toxicon, 55: 343352. DOI: 10.1016/j.toxicon.2009.08.009

Rocha, M.M.T. and M.F.D. Furtado, 2007. Análise das atividades biológicas dos venenos de Philodryas olfersii (Lichtenstein) e P. patagoniensis (Girard) (Serpentes, Colubridae). Revista Brasileira de Zoologia, 24: 410-418. DOI: 10.1590/S010181752007000200019

Sherwood, E.R. and T. Toliver-Kinsky, 2004. Mechanisms of the inflammatory response. Best Pract. Res. Clin. Anaesthesiol., 18: 385-405. DOI: 10.1016/j.bpa.2003.12.002

Teixeira, C.F.P., E.C.T. Landucci, E. Antunes, M. Chacu and Y. Cury, 2003. Inflammatory effects of snake venom myotoxic phospholipases A2. Toxicon, 42: 947-962. DOI: 10.1016/j.toxicon.2003.11.006 